\title{
Imaging Probes Offer New Opportunities for Understanding Cancer
}

\author{
Lehui Lu ${ }^{1}$ \\ Published online: 18 April 2018 \\ (c) The Nonferrous Metals Society of China 2018
}

Imaging analysis holds great promise for improving our understanding of the in vivo behavior of cancer, since it can offer important information regarding the activity of cancerrelated biomarkers and their dynamics as a function of time and space. Recently, important advances have been made in this field toward imaging probes. This thematic special issue on "Imaging Analysis" highlights recent achievements by researchers who achieved great improvement in designing novel probes for bioimaging applications.

Reactive oxygen species (ROS) play an important role in many critical physiological processes. Ma et al. present the representative spectroscopic probes for the common ROS in their review. The semiconducting polymer dots (Pdots) was used as an imaging probe by Wu et. al. By further functionalization of Pdots with holo-transferrin human (Tf), a highly efficient endocytosis process has been achieved through transferrin receptors (TfRs)-mediated internalization. Ding and coworkers review the utilization of graphene quantum dots as imaging probes. They summarize the chemical synthesis and optical properties of graphene quantum dots, and then place emphasis on in vivo imaging applications. The successful applications of surface-enhanced Raman scattering (SERS) for non-invasive biomarker detection and bioimaging offer significant clinical information to improve the diagnostic and prognostic outcomes. Wang et al. describe the case studies in their review in which different types of biomarkers have been investigated, as well as outline future challenges that must be addressed. Imaging probe-guided cancer therapy is discussed by Jiang et al. They prepare methylene blue-bovine serum albumin-Fe(III) nanoparticles (MB-BSA-Fe(III) NPs). Such nanoprobes exhibit $\mathrm{T}_{2}$ magnetic resonance imaging and excellent catalytic performance toward abundant $\mathrm{H}_{2} \mathrm{O}_{2}$ to generate $\mathrm{O}_{2}$ in cancer cells, overcoming tumor hypoxia.

I thank all the authors in this special issue on "Imaging Analysis". This series of papers will inspire the development of novel imaging probes for the better understanding of the molecular basis of cancer.

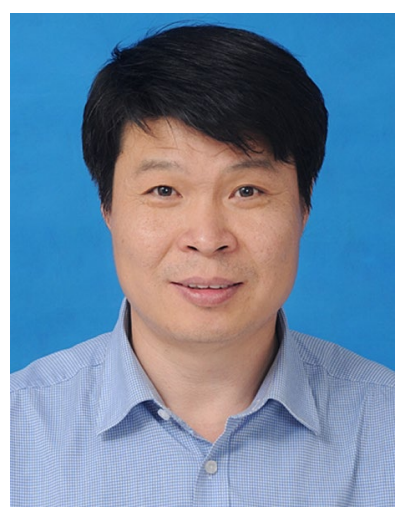

Lehui Lu received his M.S. (2000) and Ph.D. (2003) from the Changchun Institute of Applied Chemistry, Chinese Academy of Sciences (CIACCAS). He carried out his postdoctoral research at Hamburg University (2003-2005) and Kwansei Gakuin University (2005-2007). $\mathrm{He}$ is currently a full professor at CIACCAS, where he is also the director of the State Key Laboratory of Electroanalytical Chemistry. His research focuses on nano-structured functional materials for energy, biomedical and environment related applications.

Lehui Lu

lehuilu@ciac.ac.cn

1 State Key Laboratory of Electroanalytical Chemistry,

Changchun Institute of Applied Chemistry,

Chinese Academy of Sciences, Changchun 130022,

People's Republic of China 